\title{
Regional Correspondents
}

\author{
Australia \\ Tony Raftery, Dept. of Physics, \\ Queensland Institute of Technology, \\ G.P.O. Box 2434, Brisbane, Queensland 4001, Australia

\section{Japan} \\ Hideo Toraya, Ceramics Research Laboratory, \\ Nagoya Institute of Technology, \\ Asahigaoka, Tajimi 507 Japan
}

\begin{abstract}
Eastern Europe
Jaroslav Fiala, Central Research Inst. SKKODA, 31600 Plzeñ, The Czech Republic

Norberto Masciocchi

Western Europe

Dipartimento di Chimica Strutturale e Stereochimica Inorganica

Universita' di Milano

20133 Milano, Italy
\end{abstract}

\section{Calendar of Meetings}

\section{4-6 March 1997}

International Seminar on Structure and Properties of Crystalline Materials. Dubna, Russia. [Contact: Dr. V. V. Sikolenko, Frank Laboratory of Neutron Physics, Joint Institute for Nuclear Research, Dubna, Moscow Region 141980 , Russia. Tel: 7 (096) 216 5096; Fax: 7 (096) 216 5884; E-mail: sikolen@nf.jinr.ru].

\section{0-12 March 1997}

5. Jahrestagung der Deutschen Gesellschaft für Kristallographie. Hamburg, Germany. [Contact: Frau I. Bauer, Mineralogisch-Petrographisches Institut, Grindelallee 48, Universität Hamburg, D-20146 Hamburg, Germany. Tel: 49 (40) 4123 2051; Fax: 49 (40) 4123 2422; E-mail: DGK97@mineralogie.uni-hamburg.de; Info: http:// www.rrz.uni-hamburg.de/mpi/DGK97].

\section{7-21 March 1997}

International Centre for Diffraction Data Annual Spring Meeting. Newtown Square, Pennsylvania, USA [Contact: Linda Shertz, International Centre for Diffraction Data, 12 Campus Boulevard, Newtown Square, PA 19073-3273, USA, Tel: 1 (610) 325 9814; Fax: 1 (610) 325 9823; E-mail: shertz@ICDD.com; Info: http://www.icdd.com].

\section{March-4 April 1997}

Spring 1997 Meeting of the Materials Research Society. San Francisco, California, USA. The 9th International Symposium on the structure and properties of magnetic ultrathin films, multilayers, and surfaces. [Contact: Materials Research Society, 9800 McKnight Road, Pittsburgh, PA 15237. Tel: 1 (412) 367 3004; Fax: 1 (412) 367 4373; E-mail: Info@mrs.org; Info: http://www.mrs.org].

\section{2-5 April 1997}

Twentieth Meeting of the Society of Crystallographers in Australia. Queenstown, Central Otago, New Zealand. [Contact: Prof. Ward Robinson, Chemistry Department, University of Canterbury, Private Bag 4800, Christchurch, New Zealand. Tel: 64 (3) 364 2465; Fax: 64 (3) 364 2110;
E-mail: w.robinson@chem.canterbury.ac.nz; Info: http:// www.canterbury.ac.nz].

\section{4-17 April 1997}

BCA97 British Crystallographic Association Annual Spring Meeting. Leeds, England, UK. [Contact: Dr. Mark Thornton-Pett, School of Chemistry, University of Leeds, Leeds LS2 9JT, UK. Tel: 44 (113) 233 6423; Fax: 44 (113) 233 6565; E-mail: marktp@chem.leeds.ac.uk; Info: http:// www.chem.leeds.ac.uk/conferences/BCA97].

\section{1-23 April 1997}

EUROMAT 97 5th Conference of the Federation of European Materials Societies. Maastricht, The Netherlands [Contact: EUROMAT 97, P.O. Box 390, NL 3330 AJ Zwijndrecht, The Netherlands. Tel: 31 (78) 619 2655, Fax: 31 (78) 619 5735; E-mail: bvm@metropolis.nl].

\section{5-28 May 1997}

EPDIC-5 Fifth European Powder Diffraction Conference. Parma, Italy [Contact: Prof. G. Artioli, Dipartimento di Science della Terra, Universita di Milano, Via Botticelli 23, I-20133 Milano, Italy. Tel: 39 (2) 2369 8320; Fax: 39 (2) 7063 8681; E-mail: epdic@iummix.terra.unimi.it; Info: http:/ /iummix.terra.unimi.it].

\section{5-21 June 1997}

ICC 97 Eleventh International Clay Conference. Ottawa, Ontario, Canada [Contact: Dr. Jeanne Percival, Geological Survey of Canada, 601 Booth Street, Ottawa, ON, K1A 0E8, Canada. Fax: 1 (613) 943 1287; E-mail: icc97@gsc.emr.ca].

\section{6-18 June 1997}

Fifth International Conference on Residual Stresses. Linköping, Sweden. [Contact: Prof. T. Ericsson, Department of Mechanical Engineering, Linköping University, S-581 83 Linkŏping, Sweden. Fax: 46 (13) 282 505; E-mail: icrs$5 @$ ikp.liu.se]. 
23-26 June 1997

Conference on Neutron Texture and Stress Analysis. Dubna, Russia. The conference language is English. [Contact: T. S. Donskova, International Department, Frank Laboratory of Neutron Physics, Joint Institute for Nuclear Research, Dubna, Moscow Region 141980, Russia. Tel: 7 (096) 216 3856; Fax: 7 (095) 975 2381; E-mail donskova@ypr.jinr.dubna.su or Dr. H.-G. Brokmier, GKSS Research Center, Max-Planck-Strasse, Geb. 3, D-21502 Geesthacht, Germany. Tel: 49 (41) 5287 1207; Fax: 49 (41) 5287 1338; E-mail: brokmeier@gkss.de].

\section{4-27 June 1997}

ISOPS5-97 Fifth International Symposium on Pharmaceutical Sciences. Ankara, Turkey [Contact: Maksu Coskun, Faculty of Pharmacy, Ankara University, Tandogan 06100, Ankara, Turkey. Tel: 90 (312) 222 0471; Fax: 90 (312) 213 1081; E-mail: ankpharm@pharmacy.ankara.edu.tr; Info: http://www.pharmacy.ankara.edu.tr].

\section{6-9 July 1997}

CRYO97 Low Temperature Microscopy and Analysis. York, England, UK. [Contact: Royal Microscopical Society, $37 / 38$ St. Clements, Oxford OX4 1AJ, UK. Tel: 44 (1865) 248 768; Fax: 44 (1865) 791 237; E-mail: rms@vax.ox.ac.uk].

\section{3-17 July 1997}

Fifth International Conference on Surface $X$-ray and Neutron Scattering. Oxford, England, UK. [Contact: SXNS Secretariat, Room 2.33 R3, ISI Facility, RutherfordAppleton Laboratory, Chilton, Didcot, Oxon OX11 7QA,UK. Tel: 44 (1235) 446 729; Fax: 44 (1235) 445 720; E-mail: c.wadley@isise.rl.ac.uk].

\section{4-18 July 1997}

ICCM-11 Eleventh International Conference on Composite Materials. Gold Coast, Queensland, Australia [Contact: Prof. Murray L. Scott, Royal Melbourne Institute of Technology, Fishermen's Bend, GPO Box 2476V, Melbourne, Victoria 3001, Australia. Tel: 61 (3) 9645 1149; Fax: 61 (3) 9645 1027; E-mail: acss@aero.rmit.edu.au; Info: http:// www.acss.aero.rmit.edu.au/iccm-11].

\section{0-25 July 1997}

ACA '97 American Crystallographic Association Annual Meeting. St. Louis, Missouri, USA [Contact: Prof. Lee Brammer, Department of Chemistry, University of Missouri/ St. Louis, 8001 Natural Bridge Road, St. Louis, MO 631214499, USA. Tel: 1 (314) 553-5345; Fax: 1 (314)-553-5342; E-mail: Lee.Brammer@umsl.edu or Program Chair Bill Stallings, Tel: 1 (314) 537 7236; Fax: 1 (314) 537 7425; E-mail: wcstal@ccmail.monsanto.com; Infor: http:// www.hwi.buffalo.edu/aca or http://www.biochem.wustl.edu/ aca97].

\section{3-8 August 1997}

Structural Chemistry Indaba II. Kruger National Park, South Africa. An international symposium, in which each of the $(\sim 20)$ invited speakers will address a different aspect of the central theme of intermolecular interactions. [Contact: Lesley Stevenson, CEE Conference Office, Post Office Box
327, University of the Witwatersrand, Johannesburg 2050, South Africa. Tel: 27 (11) 716 5091; Fax: 27 (11) 716 7835; E-mail: indaba-org@hobbes.gh.wits.ac.za; Info: http:// www.gh.wits.ac.za/indaba].

\section{4-8 August 1997}

46th Annual Denver X-ray Conference. Steamboat Springs, Colorado, USA [Contact: Paul Predecki or Carole Collier, Department of Engineering, University of Denver, Denver, CO 80208, USA. Tel: 1 (303) 871 3515; Fax: 1 (303) 871 3515; Fax: 1 (303) 871 4450; E-mail: denxrcon@du.edu; Info: http://www.engr.du.edu].

\section{4-8 August 1997}

Fourth Annual Symposium on High Resolution Diffraction and X-ray Topography. Steamboat Springs, Colorado, USA. Held within the 46th Ammia; Demver X-ray Conference. [Contact: see above.]

\section{August 1997}

International Centre for Diffraction Data Technical Meetings. Steamboat Springs, Colorado, USA [Contact: Linda Shertz, International Centre for Diffraction Data, 12 Campus Boulevard, Newtown Square, PA 19073-3273, USA. Tel: 1 (610) 325 9814; Fax: 1 (610) 325 9823; E-mail: shertz@ICDD.com; Info: http://www.icdd.com].

\section{7-21 August 1997}

ICNS 97 International Conference on Neutron Scattering. Toronto, Ontario, Canada. [Contact: Dr. Phyllis H. Green, Solid State Division, Oak Ridge National Laboratory, Post Office Box 2008, Oak Ridge, TN 37831-6033, USA. Tel: 1 (423) 576 1864; Fax: 1 (423) 574 4143; E-mail: phg@ornl.gov or Thom Mason, Department of Physics, University of Toronto, 60 St. George Street, Toronto, ON Canada M5S 1A7. Tel: 1 (416) 978 7868; Fax: 1 (416) 978 2537; E-mail: mason@swelter.physics.utoronto.ca; Info: http://www.physics.utoronto.ca/icns/icns.htm1].

\section{7-21 August 1997}

OCC 97 Tenth Symposium on Organic Crystal Chemistry. Rydzyna (near Poznan), Poland [Contact: Prof. Urszula Rychlewska, Faculty of Chemistry, Adam Mickiewicz University, Grunwaldzka 6, 60-780 Poznan, Poland. Tel: 48 (61) 699181 ext 489; Fax: 48 (61) 658 008: E-mail: occ97@plpuam11.amu.edu.pl; Info: http:// www.man.poznan.p1/ occ97].

\section{4-27 August 1997}

International Symposium on Zeolites and Microporous Crystals. Tokyo, Japan. [Contact: Takashi Tatsumi, Engineering Research Institute, University of Tokyo, 2-11-16 Yayoi, Tokyo 113, Japan. Tel: 81 (3) 38122111 ext. 7705; Fax: 81 (3) 5800 6825; E-mail: tatsumi@komiyama.t.utokyo.ac.jp].

\section{4-28 August 1997}

ECM-17 European Crystallographic Meeting. Instituto Superior Técnico. Lisboa, Portugal [Contact: ECM-17 Conference Secretariat, Attn: Alexandra Azevedo, IBET, Apartado 12, P-2780 Oeiras, Portugal. Tel: 351 (1) 7823; Fax: 351 (1) 443 3644; E-mail: ecm17@itqb.unl.pt; Info: http://alfa.ist.utl.pt]. 
25-29 August 1997

ICAMS-17 17th International Conference on Amorphous and Microcrystalline Semiconductors. Budapest, Hungary. [Contact: P. Barna, Research Institute for Technical Physics, Post Office Box 76, Budapest H-1325, Hungary. E-mail: barna@mufi.hu].

\section{7-31 August 1997}

APERIODIC '97 International Conference on Aperiodic Crystals. Palais des Sports et des Congres, Alpe d'Huez (close to Grenoble), France [Contact: Aperiodic ' 97 Secretariat, Attn, Mme. B. Aubert, Institut Laue-Langevin, BP 156, 38042 Grenoble Cedex 9, France, Tel: 33 (04) 7620 7008, Fax: 33 (04)7648 3906; E-mail: aprd97@ill.fr; Info: http://www.inpg.fr/ltpcm/aprd97/aprd97_1.html].

\section{August-4 September 1997}

17th Conference on Applied Crystallography. WislaJawornik, Poland. [Contact: Prof. H. Morawiec or Dr. Danuta Stroz, Institute of Physics and Chemistry of Materials, University of Silesia, Bankowa 12, 40-007 Katowice, Poland. Tel: 48 (32) 596 929; Fax: 48 (32) 599 605; E-mail: dana@uscrouxl.cto.us.edu.pl].

\section{1-26 September 1997}

Second International Symposium on Structural Intermetallics. Champion, PA USA. [Contact: M. Nathal, NASA Lewis Research Center, 21000 Brookpark Road, MS 106-5, Cleveland, OH 44135. E-mail: michael.v.nathal@lerc.nasa.gov].

\section{1-5 December 1997}

Fall 1997 Meeting of the Materials Research Society. Boston, MA, USA. [Contact: Materials Research Society, 9800 McKnight Road, Pittsburgh, PA 15237. Tel: 1 (412) 367 3004; Fax: 1 (412) 367 4373; E-mail: info@mrs.org; Info: http://www.mrs.org].

\section{3-17 April 1998}

Spring 1998 Meeting of the Materials Research Society. San Francisco, CA, USA. [Contact: Materials Research Society, 9800 McKnight Road, Pittsburgh, PA 15237. Tel: 1 (412) 367 3004; Fax: 1 (412) 367 4373; E-mail: info@mrs.org; Info: http://www.mrs.org].

\section{May 1998}

EPDIC-6 Sixth European Diffraction Conference. Budapest, Hungary.
10-14 August 1998

IMA'98 17th General Meeting of the International Mineralogical Association. Toronto, Ontario, Canada. The meeting will be centered on extensive scientific sessions, preceded and followed by field trips across Canmada and the US including important mineral locations, ore deposits, sites of impact craters, and classic petrologic locations. [Contact: Dr. Eva Schandl, Department of Geology, University of Toronto, Earth Sciences Centre, 22 Russell Street, Toronto, ON M55 3B1, Canada. Tel: 1 (416) 478 7084; Fax: 1 (416) 478 3938; E-mail: ima98@quartz.geolog.utoronto.ca; Info: http:/ /www.geology.utoronto.ca/IMA98]

\section{6-22 August 1998}

ECM-18 18th European Crystallographic Meeting. Prague, Czech Republic. [Contact: E-mail: hasek@imc.cas.cz].

\section{9-24 August 1998}

ACA '98 American Crystallographic Association Annual Meeting. Washington, DC, USA. [Contact: T. A. Vanderah, National Institute of Standards and Technology, Ceramics Division, Building 223 Room A256, Gaithersburg, MD 20899, USA. Tel: 1 (301) 975 5785; Fax: 1 (301) 9908729 ; E-mail: terrell@credit.nist.gov or V. L. Karen, NIST, Materials Science and Engineering Laboratory, Materials Building Room A215, Gaithersburg, MD 20899, USA. Tel: 1 (301) 975 6255; E-mail: karen@tiber.nist.gov; Info: http:// www.hwi.buffalo.edu/aca].

\section{May 1999}

ACA '99 American Crystallographic Association Annual Meeting. Buffalo, New York, USA [Info: http:// www.hwi.buffalo.edu/aca].

\section{4-13 August 1999}

18th IUCr General Assembly and International Congress of Crystallography. Glasgow, Scotland, UK [Contact: Dr. C. J. Gilmore, Department of Chemistry, University of Glasgow, Glasgow G12 8QQ, Scotland. Tel: 44 (141) 339 8855, Fax: 44 (141) 330 4418; E-mail: iucr@ chem.gla.ac.uk or Prof. J. A. K. Howard, University of Durham, Department of Chemistry, South Road, Durham DH1 3LE, England, UK. Tel: 44 (191) 374 4647, Fax: 44 (191) 374 3745; E-mail: j.a.k.howard@durham.ac.uk; Info: http://www.chem.gla.ac.uk/iucr99].

\section{Regional Reports}

A Report on the Poster Session of the ICDD October 1996 Technical Meetings. Hideo Toraya, Ceramics Research Laboratory, Nagoya Institute of Technology, Asahigaoka, Tajimi 507, Japan

The poster session was held during the ICDD October 1996 Technical Meetings. The theme of the session was "Materials Characterization by X-ray Analysis Techniques." There were a total of five posters, displayed outside the conference rooms during the meetings. Topics include the diffraction study of crystallization process of poly (ethylene-2,6-naphthalate), the quantitative phase analysis of up to ten components mixtures using whole-powder-pattern decomposition technique, the $a b$ initio structure determinations using computational chemistry techniques and Rietveld refinement, Rietveld refinements of a series of $\mathrm{Ba}-\mathrm{Sr}$ lanthanide superconductor materials, and the ICDD/PDF su- 